\title{
An Approach for Automatic Learning and Inference by Knowledge Map
}

\author{
Chang-Kai Hsu, Jyh-Cheng Chang, Maiga Chang, Jihn-Chang Jehng and Jia-Sheng Heh
}

ken@mcsl.ice.cycu.edu.tw, eoda@mcsl.ice.cycu.edu.tw, maiga@ms2.hinet.net,

\author{
jehng@src.ncu.edu.tw, jsheh@ice.cycu.edu.tw \\ Dept. of Information Computer and Engineering, \\ Chung-Yuan Christian Univ., Chung-Li, 320, Taiwan
}

\begin{abstract}
Knowledge is represented by graph or map is simpler than represented by proposition logics. Students who use the knowledge representation of knowledge map quickly learn to read a graph as $I F-T H E N$ and do all their reasoning directly on the graphs without translating them to rules or propositions. Instead, many graphic notations have been developed. Knowledge Map (KM) is a graphic system of logic that is as general as proposition logics.
\end{abstract}

Keywords: knowledge representation, conceptual graph, knowledge map.

\section{Introduction}

Knowledge representations that use some notations or expressions, such as text, table or graph, to represent knowledge had been researched many years by researchers. According to these representations, the graph is more readable and realizable by human than others because it more simper and more intension to represent. [CHO93]

Knowledge Map were designed to have a direct mapping to and from structured documents; in addition, to use the knowledge map be knowledge representation for problem solving can also be an application in computer system. [HaF94]

\section{Knowledge Formulation}

Content structures are used to determine the sequence and content of instructions to promote understanding of the author's perspective on the content area. Content structures are constructed from the sentences, denoted by $p_{m}$, which always have some topic or subtopics as Figure 1 shown below.[Nict99]

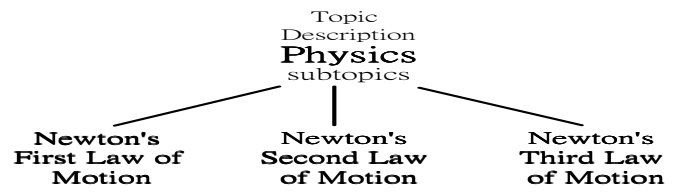

Figure 1. Content structure

Concept $\theta_{i}$ is the basic unit of knowledge used to represent something. The concept space, $\underline{\theta 0}=\left\{\theta_{i}\right\}$, represents the discourse of all the concepts, such as all students in this school or all keywords in Physics. A concept set, denoted as $\underline{\theta}=\left\{\theta_{i}\right\} \subseteq \underline{\theta 0}$, is one subset of concepts.

A concept relation, represented as $\rho_{k}\left(\theta_{i 1}, \theta_{i 2}, \ldots\right)$, is an aggregation (relation) of knowledge pieces (concepts $\theta_{i 1}, \theta_{i 2}, \ldots$ ) with some specific relation style $\rho_{k}(\cdot)$. The concept relation space, symbolized as $\underline{\varrho 0}=\left\{\rho_{k}\right\}$, is the discourse of all the concept relation.

Formally, a proposition can be represented as $\phi_{k}\left(\rho_{j}\left(\theta_{i 1}, \theta_{i 2}, \ldots\right)\right)$, mapping a concept relation $\rho_{j}\left(\theta_{i 1}, \theta_{i 2}, \ldots\right)$ into a value $\phi(\cdot) \in V_{\phi}$. The value set $V_{\phi}$ adopts usually true-false logic, and can be other data types such as fuzzy sets or continuous values. The proposition space, $\underline{\phi 0}=\left\{\phi_{k}\right\}$, represents the discourse of all the proposition, such as all statements or valus in Physics. A proposition set, denoted as $\underline{\phi}=\left\{\phi_{k}\right\}$ 
$\subseteq \underline{\phi 0}$, is one set of propositions.

\section{Analysis of Knowledge Hierarchical}

The general knowledge unit, denoted as $K U_{p}\left(\theta_{i}\right.$ $\Phi)$, that includes the concept and all its propositions (knowledge schemas). And each proposition maps a concept relation and its concepts. For instance, the concept relations in the propositions about bird are

"BIRD can FLY" $=\rho_{\text {CAN }}\left(\theta_{\text {BIRD }}, \theta_{\text {MAMMAL }}\right)$, and

"BIRD has WINGS" $=\rho_{\text {HAS_A }}\left(\theta_{\text {BIRD }}, \theta_{\text {WINGS }}\right)$ etc.

The relations of concepts inside a knowledge unit, $\rho_{\text {INTERPRETATION }}\left(\theta_{\text {SIGNIFIER }}, \theta_{\text {SIGNIFIED }}\right)$. The propositions of those knowledge units' concept relations can also express:

- production rule,

$\rho_{I F_{-} T H E N}\left(\rho_{I F}\left(\theta_{1}, \theta_{2}, \ldots\right), \rho_{\text {THEN }}\left(\theta_{l}, \theta_{2}, \ldots\right)\right)$

- concept schema

$$
\begin{gathered}
\rho_{\text {SCHEMA }}\left(\theta_{\text {OBJ }}, \theta_{\text {ATTRI }}, \theta_{A T T R 2}, \ldots\right)= \\
\rho_{\text {SCHEMA }}\left(\theta_{\text {OBJ }}, \rho_{\text {LINK }}\left(\theta_{\text {OBJ }}, \theta_{\text {ATTRI }}\right),\right. \\
\left.\rho_{\text {LINK }}\left(\theta_{\text {OBJ }}, \theta_{\text {ATTR2 } 2}\right), \ldots\right)
\end{gathered}
$$

- formula,

$$
\rho_{\text {FORMULA }}\left(\theta_{\mathrm{X} 1}, \theta_{\mathrm{X} 2}, \theta_{\mathrm{Y}}, \ldots\right)
$$

- concept hierarchy, syntagm, $\rho_{I S \_A}\left(\theta_{1}, \theta_{2}\right), \rho_{H A S_{-} A}\left(\theta_{1}, \theta_{2}\right) \ldots$. etc.

If $C G\left(\underline{\theta}, \underline{\rho}_{\text {CLUSTER }} \cup \underline{\rho}_{\text {LINKAGE }}\right)$ is a lattice, then it is called $K H\left(\underline{\theta}, \underline{\rho}_{K I N D} \cup{\underline{\rho_{P A R T}}}\right)$.

- Concept intension, $\operatorname{specify}\left(\theta_{\mathrm{i}}\right)$

$$
\operatorname{specify}\left(\theta_{\mathrm{i}}\right)=\left\{\theta_{\mathrm{j}}: \operatorname{level}\left(\theta_{\mathrm{j}}\right)=\operatorname{level}\left(\theta_{\mathrm{i}}\right)+1\right. \text { and }
$$

$$
\left.\rho_{\mathrm{k}}\left(\theta_{\mathrm{i}}, \theta_{\mathrm{j}}\right), \rho_{\mathrm{k}} \in \underline{\rho}_{\text {CLUSTER }}\right\}
$$

- Concept extension, generalize $\left(\theta_{\mathrm{i}}\right)$ generalize $\left(\theta_{\mathrm{i}}\right)=\left\{\theta_{\mathrm{j}}: \operatorname{level}\left(\theta_{\mathrm{j}}\right)=\operatorname{level}\left(\theta_{\mathrm{i}}\right)-1\right.$ and

$$
\left.\rho_{\mathrm{k}}\left(\theta_{\mathrm{j}}, \theta_{\mathrm{i}}\right), \rho_{\mathrm{k}} \in \underline{\rho}_{\text {CLUSTER }}\right\}
$$

\section{3-stage learning algorithm for constructing}

\section{knowledge map}

The knowledge map is learning from sentences that got from text/html, if some concepts, relations and propositions are defined.

Algorithm 1: Learning algorithm
Step 1: Select the main topic for the knowledge map and store it in the root knowledge units.

Step 2: Decomposition proposition to a number of relations.

Step 3: Categorize the concepts, grouping similar concepts and labeling the groups.

Composition or decomposition of two knowledge maps:

Algorithm 2: (De)Composition algorithm

Step 1: Union the concept space.

Step 2: Removing the redundant concepts if they exist.

Step 3: Removing the transitive relation and multi-relation if they exist.

\section{Discussion and Conclusion}

In this paper, one knowledge representation method is discussed, especially for structured document. Many problems in other fields, which are procedural problem in the mathematics, may also be solved by Knowledge Map (KM). Figure 5. A Knowledge Map from website.

\section{References}

[CHO93] Gary Chartrand and Ortrud R. Oellermann, Applied and Algorithmic Graph Theory, McGraw-Hill, Inc., pp. 58-60, 1993

[HaF94] Jiawei Han and Yongjian Fu, "Dynamic Generation and Refinement of concept Hierarchies for Knowledge Discovery in Databases", Proc. AAAI'94 Workshop on Knowledge in Databases, 1994

[Nict99] Physics and Chemical Textbook for Junior High School, National Institute for Compilation and Translation, Taipei, Vol.2, Section 8-1, pp.20-25, 1999 (in Chinese) 